\title{
Multi-attribute decision-making based on soft set theory: a systematic review
}

\begin{abstract}
Multi-attribute decision-making (MADM) is a cognitive process for evaluating data under various attributes in order to find the best option in terms of the preferences of decision makers. Handling uncertainty and vagueness in most real-world decision-making problems involves the application of mathematical tools dealing with uncertainty, such as soft set (SS) theory in decision-making processes. In recent years, different approaches have been suggested for demonstrating the effectiveness and efficiency of soft set theory in MADM. To date, no systematic literature review has been presented in this area. However, a review paper on existing SS-based methods can help future researchers to find the gaps and missing MADM approaches in current studies. In this paper, we review the methodologies and applications of 71 research papers published in 30 academic journals (extracted from online databases including ScienceDirect, Scopus, Springer, World of Scientific, and Hindawi) between January 2000 and December 2017. These papers have been categorized into two main groups: single and hybrid approaches. In addition, they have been classified with respect to MADM techniques, country of origin, journal, and publication year. The statistical analysis shows that "SSAW" method which has been employed by various authors is the most common technique. Moreover, the number of research publications by academics was highest in 2017. In addition, the "Applied Mathematical Modelling" journal and China are ranked as the academic journal and country that have presented the most major contributions in this domain, respectively.
\end{abstract}

Keyword: Soft set; Fuzzy soft set; Multi-attribute decision-making; Literature review 